\# GOETHE INSTITUT TEMPORAL, PROVIDENCIA, CHILE

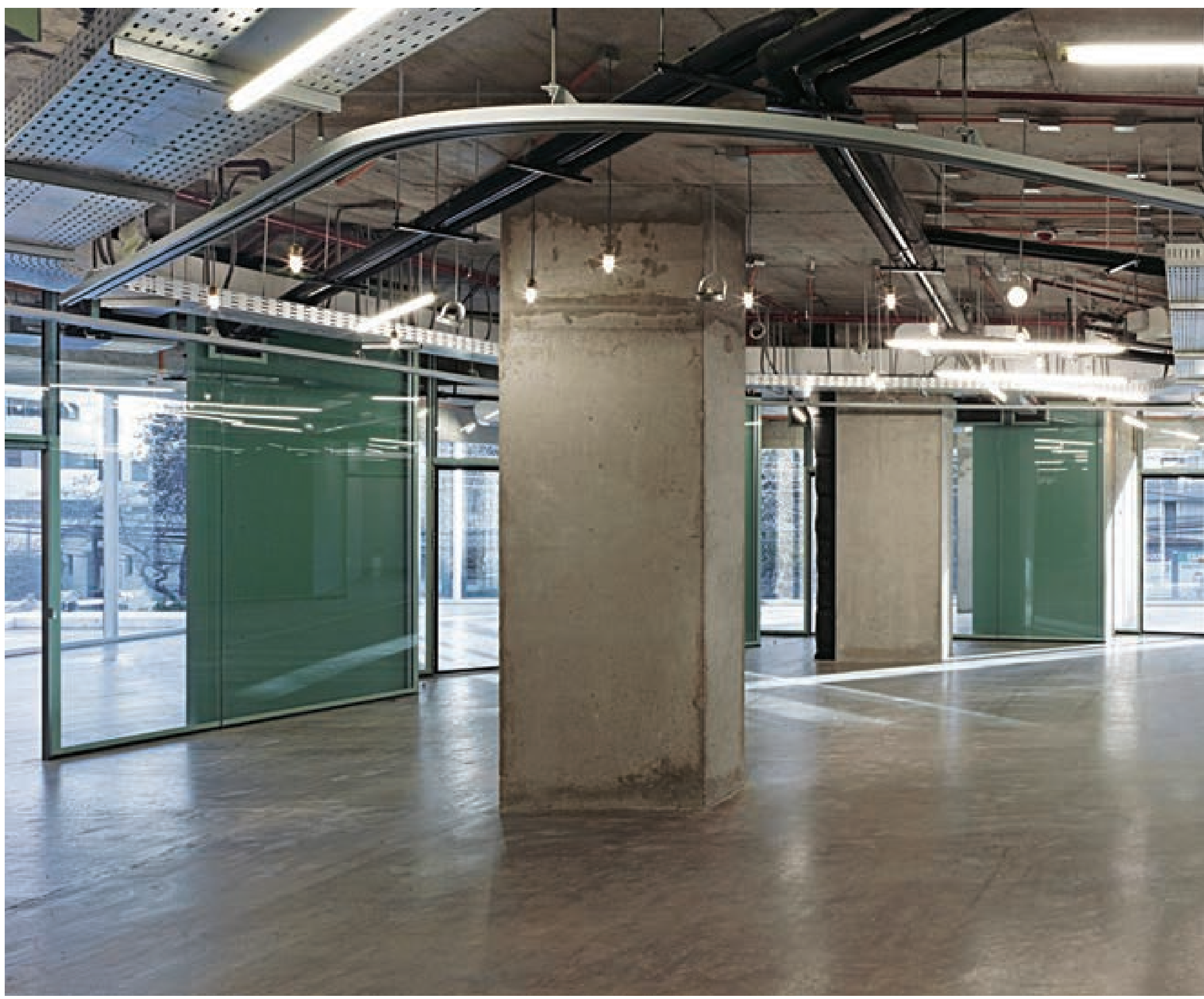

Marc Frohn

Profesor, Royal College of Art, Londres

Arquitecto, Universitá degli Studi Federico II, 1999; Máster en Arquitectura, University of Houston, 2001 y Rice University, 2004. Ha colaborado con OMA / Rem Koolhaas y con

b\&k+brandlhuber\&co. Ha sido profesor en RWTH - Aachen,

Alemania y en SCl-arc, EE.UU. Desde el año 2009 es profesor en el Royal College of Art en Londres.

\title{
Mario Rojas
}

Profesor, Universidad Andrés Bello, Santiago

Arquitecto, RWTH - Aachen, 2002. Ha colaborado con GMP

Architekten, Oscar Niemeyer y bgp de Bernardo Gómez-Pimienta. En 2004 junto a Marc Frohn fundó FAR FROHN \& ROJAS, con sedes en en Berlín, L.A. y Santiago. Desde el año 2007 es profesor de la Universidad Andrés Bello. 
FAR FROHN \& ROJAS, 2010-2011

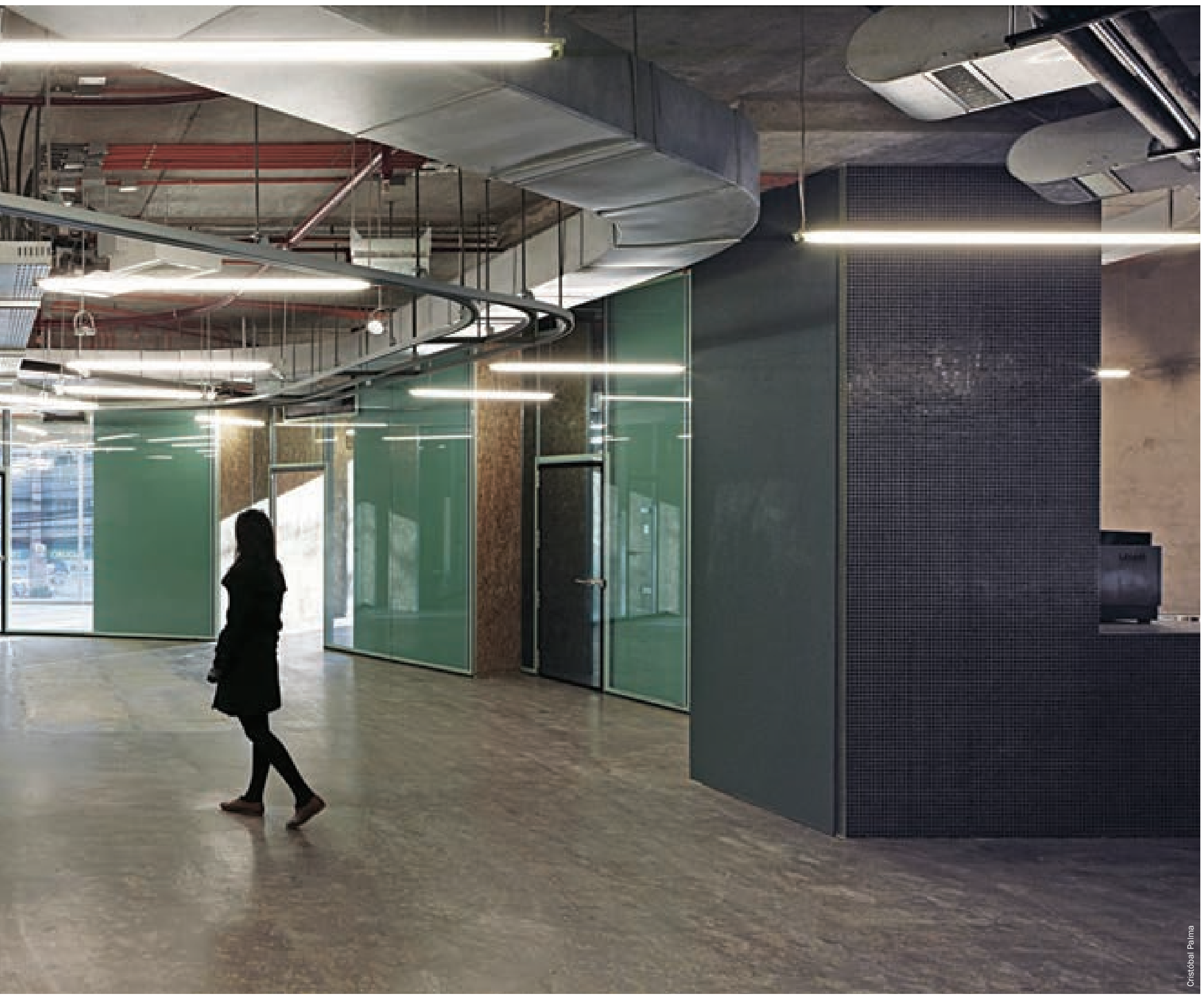

Para la instalación transitoria de un instituto binacional en la planta libre de un edificio de oficinas, este proyecto de arquitectura despliega un repertorio material que incluye textiles, mosaicos, vidrio y carpinterías de madera: a través de la organización de estos elementos se consigue un espacio interior que, con ligereza, admite diversas reconfiguraciones, permanentemente atravesado por la luz natural que entra desde la fachada vidriada

PALABRAS CLAVE Arquitectura-Chile, arquitectura alemana, centro cultural, espacios flexibles, planta libre

A bi-national cultural institution move temporarily to a typical of fice floor plan; the project of architecture introduces a collection of textiles, mosaics, glass and woodwork to house it. The layout delivers a well-lit, flexible space that can be reconfigured at ease, ensuring the entrance of sunlight from the glazed façade.

KEYWORDS Architecture-Chile, German architecture, cultura center, flexible space, open floor plan 
El edificio que históricamente había alojado las dependencias del Goethe Institut en la calle Esmeralda, en el centro de Santiago, resultó dañado tras el terremoto de febrero de 2010 . Luego de un concurso de arquitectura, FAR fue el estudio designado para desarrollar un proyecto de renovación para la estructura; sin embargo, era necesario implementar una solución temporal mientras duraban los trabajos de reparación.

La sede transitoria del instituto se instaló en un piso sin terminaciones en un edificio de oficinas en Providencia. Una crujía -quizás demasiado profunda- de $16 \mathrm{~m}$ se definía entre el núcleo de circulaciones y la fachada vidriada. Pero a pesar de estas magnitudes, el espacio se hacía escaso para acomodar salas de clases, biblioteca, oficinas y las áreas de exposición y actividades sociales.

La amplia planta libre fue subdividida con un repertorio de piezas que combina muebles de catálogo existentes y un sistema nuevo de estantes metálicos, que configuran el lenguaje de componentes del proyecto. La estrategia minimiza los recursos empleados y al mismo tiempo genera un paisaje interior consistente. Las divisiones realizadas con el mobiliario se ubican radialmente, permitiendo vistas largas hacia el exterior desde cualquier punto y abriendo el paso de la luz natural hacia lo más profundo de la planta. Esa disposición radial del conjunto de servicios, expuestos y coloridos, enfatiza la lógica centrífuga del esquema.

A lo largo de la fachada se han atenuado los límites espaciales entre oficinas y salas de clases. De la misma forma, hacia el interior de la planta se minimizan las distinciones entre las áreas de biblioteca y exposición. Una pesada cortina aislante permite una separación acústica eficiente, pero flexible, entre el área de actividades sociales y una de las mayores aulas; mientras una membrana curva y continua de metal desplegado separa la biblioteca de las oficinas administrativas más privadas y genera inesperados vínculos visuales entre ambos sectores.

La nueva sede del Goethe Institut puede ser reconfigurada con soltura de acuerdo a las actividades y demandas que en ella tengan lugar en el curso del día. Materiales y superficies que emiten luz añaden por la noche una misteriosa capa extra de información en el interior. Los mismos componentes proyectuales de este escenario temporal encajarán perfectamente en la renovación del edificio histórico, donde tendrán otra lectura en el espacio. ARQ

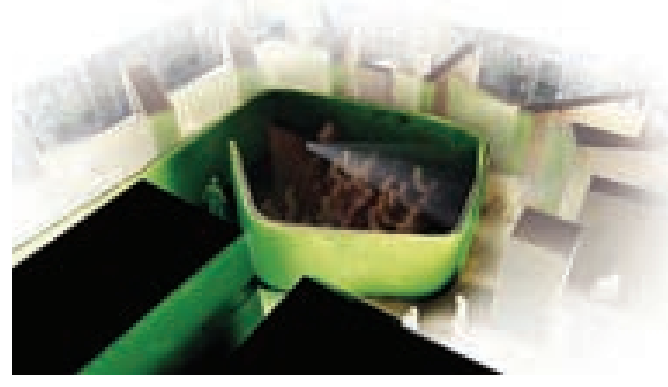

Área de eventos - pantalla de proyección Cortina acústica cerrada

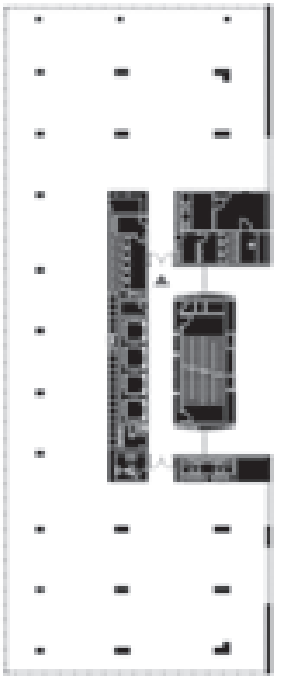

Piso tipo existente - oficinas E. 1: 1.000

$$
\begin{aligned}
& \text { : }
\end{aligned}
$$

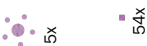

$$
\begin{aligned}
& \text { " 㻌希 } \\
& \text { " " }
\end{aligned}
$$

Inventario de mobiliario existente

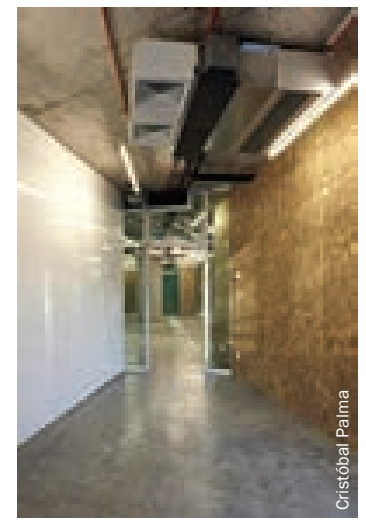

Sala de clases

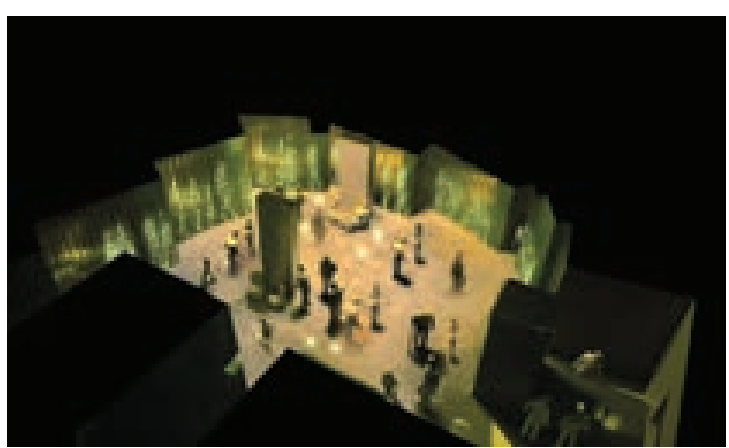

Área de eventos - recepción nocturna

Marcos fluorescentes de borde, iluminación interior suave

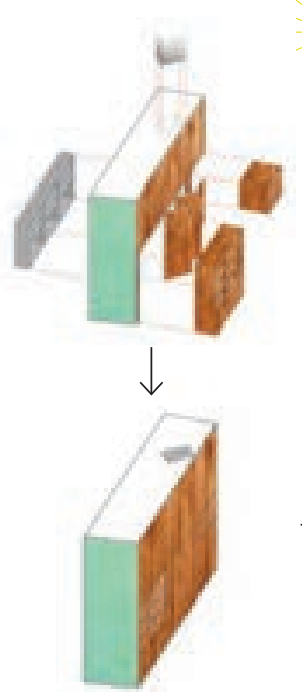

Módulos de mobiliario basados

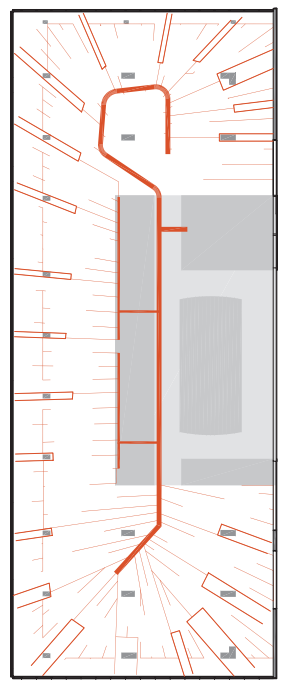

Redes de electricidad E: 1: 1.000

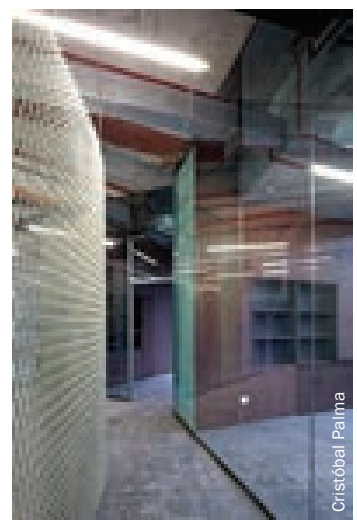

Corredor entre biblioteca y oficinas en los armarios existentes

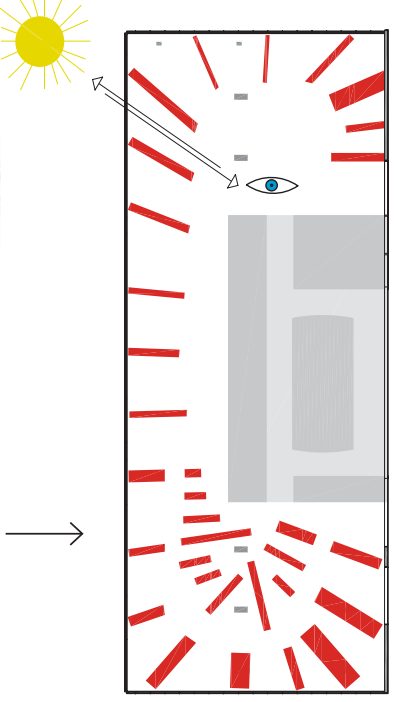

Muros de almacenamiento E. 1: 1.000

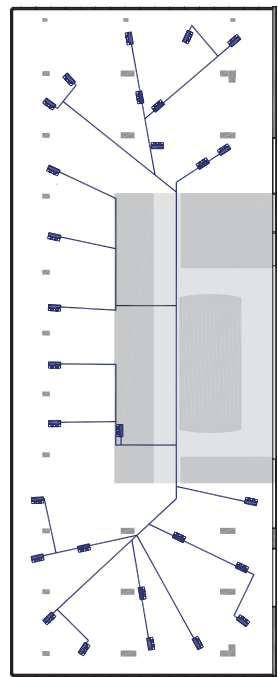

Red de enfriamiento E. 1: 1.000

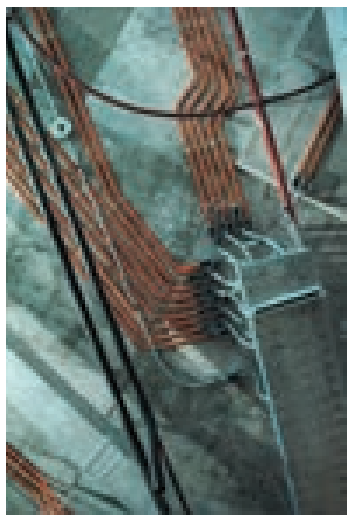

Instalaciones técnicas en cielo

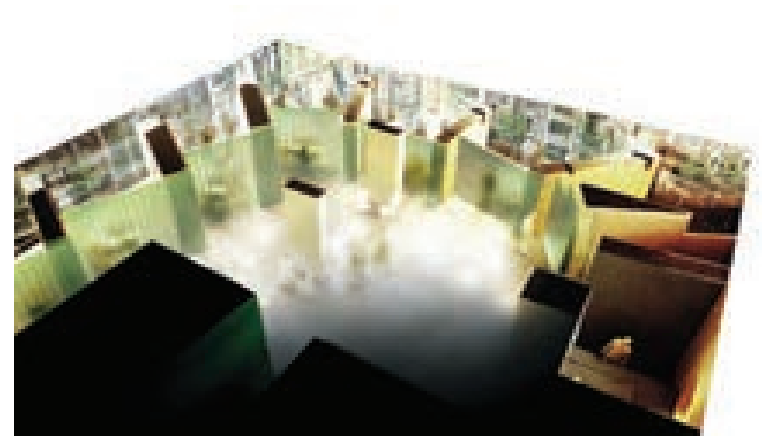

Área de eventos - talleres de lengua alemana Vistas abiertas hacia el exterior 


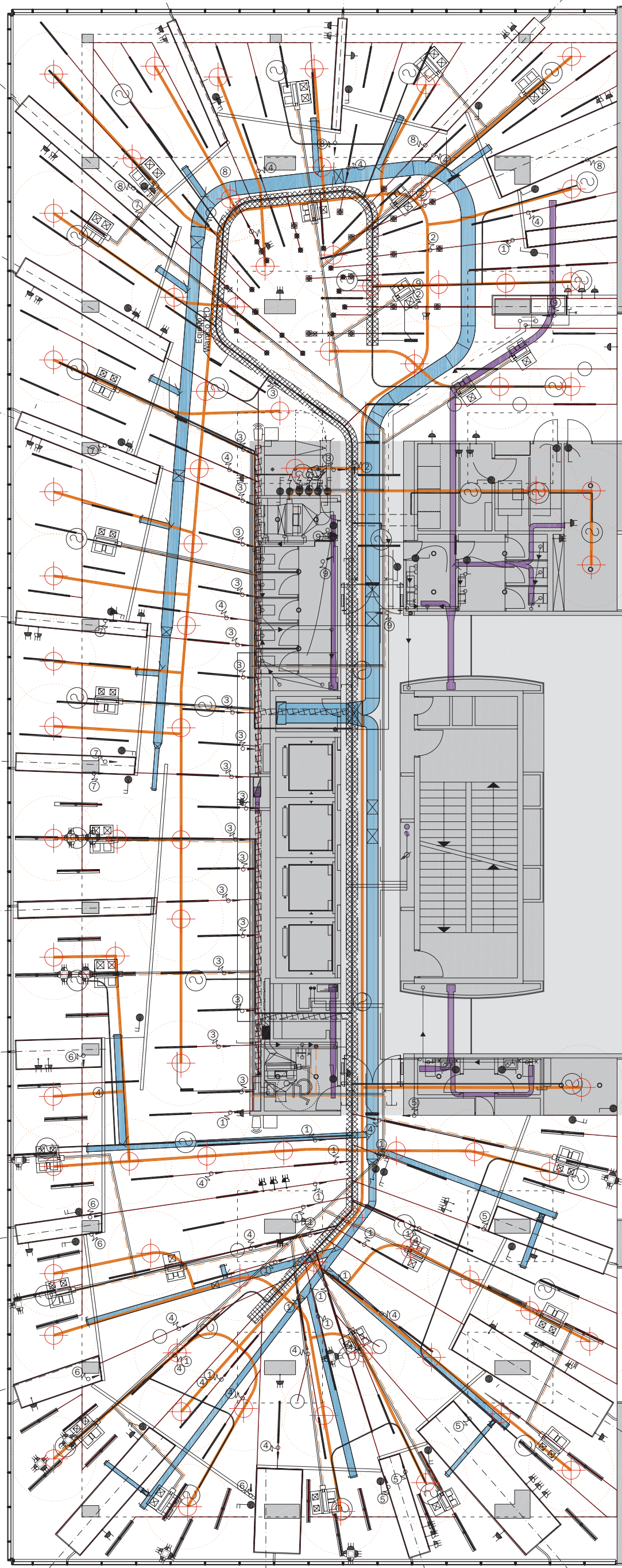

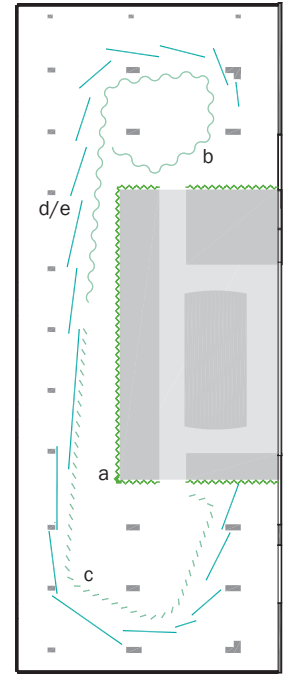

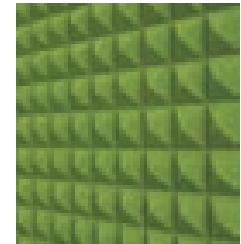

Distribución de membranas divisorias E. 1: 1.000

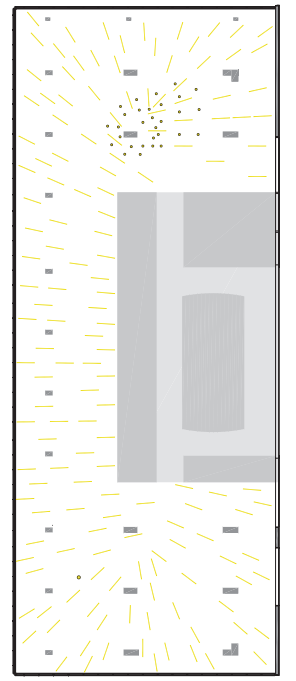

lluminación ^ E. 1: 1.000

Red de aire acondicionado

E. 1: 1.000

Proyección de planta de cielo

E. 1: 250

1. Cocina

2. Servicios

3. Sala de clases

4. Área de eventos

5. Sala de estudios

6. Sala de profesores

7. Oficina

8. Sala de reuniones

9. Biblioteca

O

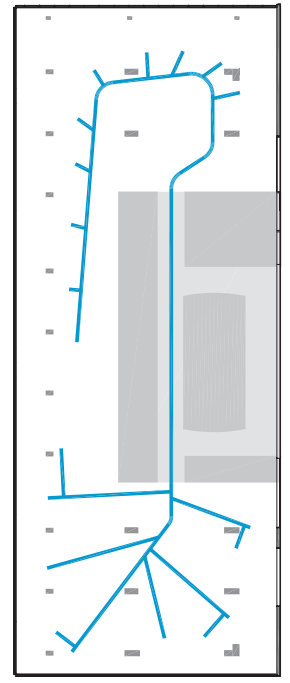

a. Espuma acústica

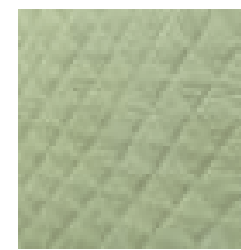

b. Cortina acústica

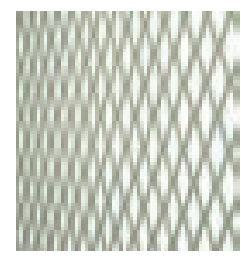

c. Membrana metal desplegado

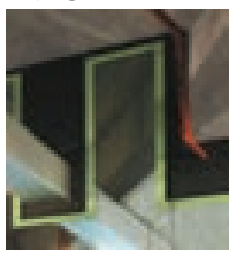

d. Cerramiento de sala de clases

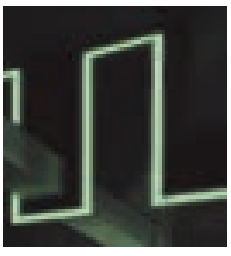

Borde fluorescente
Goethe Institut Temporal / Arquitectos: FAR FROHN \& ROJAS. Marc Frohn, Mario Rojas Toledo / Colaboradores: Max Koch, Natalia Becerra, Fabio Magnago, Steven Vidovic Tim Maaßen, Philipp Kentgens, Marius Helten, Isabel Miño, Pia Custodis, Alex Seick, Reina Pisano, Nikola Freissmuth / Ubicación: avenida Holanda, Providencia, Santiago / Cliente: Gobierno de la República Federal de Alemania / Construcción: Constructora Ralún Ltda. / Proyecto sanitario: Constanzo EIRL / Proyecto eléctrico: PRoINGEL Ltda. / Proyecto de iluminación: Docevolts / Proyecto de seguridad: Grupo Schutz S.A. / Climatización: Masterclima S.A. / Proyecto de redes de datos: Redes integradas DECOM Ltda. / Instalaciones especiales: Constanzo EIRL (malla de metal desplegado, riel de cortina acústica, puertas cortafuego); Alumcrist Ltda. (cristales), Creizet S.A. (pavimentos), Luminotecnia S.A. (luminarias); Muebles Pirámide Ltda. (carpinterías en madera); Puertas Bunker S.A. (puertas cortafuego); M3MBRANE Sails (cortina acústica) / Materiales predominantes: panel absorbente acústico con estructura piramidal de poliuretano de celda abierta, metal desplegado lacado, cortina acústica de vinilo, lana de vidrio suede textil como revestimiento, cristal laminado de color de $16 \mathrm{~mm}$ tableros OSB y postformados diversos / Superficie construida: $1.024 \mathrm{~m}^{2}$ / Año del proyecto: 2010 / Año de construcción: 2011 / Fotografía: Cristóbal Palma, archivo de FAR FROHN \& ROJAS

Bibliografía sugerida

FROHN, Marc y Mario ROJAS. “Wall House”. ARQ № 76, Día y noche. Ediciones ARQ, Santiago, diciembre de 2010, pp. 28-35. ELSER, Oliver. Deutsche Architektur Jahrbuch

German Architecture Annual, 2008-2009. Deutsches

Architekturmuseum - Prestel, Frankfurt am Main, diciembre de 2009

http://www.f-a-r.net/ 